\title{
COMUNICAÇÃO
}

\section{COMPARAÇÃO ENTRE MODELOS PARA PREDIÇÃO DO NITROGÊNIO MINERALIZADO: UMA ABORDAGEM BAYESIANA}

\author{
Comparison of the models for prediction of the mineralized nitrogen: a bayesian approach
}

\author{
Janser Moura Pereira ${ }^{1}$, Joel Augusto Muniz², Thelma Sáfadi ${ }^{3}$, Carlos Alberto Silva ${ }^{4}$
}

\begin{abstract}
RESUMO
Neste trabalho, desenvolveu-se uma abordagem bayesiana para predizer as quantidades de nitrogênio mineralizados por intermédio de modelos não lineares. Os modelos não lineares considerados para avaliar a dinâmica da mineralização do nitrogênio e para ilustrar o procedimento bayesiano foram: modelo de Cabrera, Marion, Stanford e Smith. A comparação dos modelos foi feita por meio do Fator de Bayes $(F B)$ e do Critério de Informação Bayesiano $(B I C)$. A inferência sobre os parâmetros realizou-se por intermédio do Amostrador de Gibbs e do Metropolis Hastings. O modelo de Cabrera (1993) foi o que proporcionou melhor qualidade de ajuste ao conjunto de dados de mineralização de nitrogênio, sendo seguido pelo modelo de Stanford \& Smith (1972) e, por último, o de Marion et al. (1981).
\end{abstract}

Termos para indexação: Amostrador de Gibbs, Metropolis Hastings, Fator de Bayes, Critério de Informação Bayesiano.

\section{ABSTRACT}

In this work one developed a Bayesian approach to predict the amount of mineralized nitrogen through nonlinear models. The nonlinear models considered to evaluate the mineralization of organic nitrogen and to illustrate the Bayesian procedure were: models of Cabrera, Marion, Stanford and Smith. The comparison of the models was promoted through the Bayes Factor (FB) and Bayes Information Criterion (BIC). Inference on the parameters was carried out through the Gibbs Sampling and Metropolis Hastings. The model that provided better adjustment quality to the group of data was Cabrera's model (1993), followed by the model of Stanford \& Smith (1972) and the last one by Marion et al. (1981).

Index terms: Nitrogen mineralization, Nonlinear models, Bayesian inference.

(Recebido em 22 de fevereiro de 2007 e aprovado em 6 de agosto de 2007)

O nitrogênio potencialmente mineralizável do solo $\left(N_{0}\right)$ é uma medida da quantidade de nitrogênio mineralizado sob condições ótimas de umidade e temperatura, podendo ser empregado para estimar o nitrogênio $(\mathrm{N})$ que será disponibilizado durante o ciclo de uma cultura. Compreender as transformações do $\mathrm{N}$ no solo constitui um fator importante para a maximização de seu uso pelas plantas, e também para uma maior eficiência na adubação nitrogenada, uma vez que o nitrogênio é essencial ao crescimento e à produção das culturas, constituindo, na maioria dos casos, no elemento que mais limita o crescimento e desenvolvimento das plantas (SILVA et al., 1994).

$\mathrm{Na}$ agricultura comercial moderna, grande quantidade de nitrogênio é adicionada ao solo, constituindo-se fonte potencial de poluição ambiental.
Embora tal contaminação seja proveniente de várias fontes, como resíduos municipais e industriais, as práticas agrícolas, em especial a adubação do solo com nitrogênio que têm aumentado abusivamente nos últimos anos, têm sido consideradas as principais delas (FERGUSON et al., 1991). Para que este problema decresça, é necessário que os agricultores usem adubos nitrogenados e orgânicos em quantidades adequadas que, ao mesmo tempo, aumentem a produtividade das culturas e diminuam ao mínimo possível os teores de $\mathrm{N}-\mathrm{NO}_{3}^{-}$(nitrato) e $\mathrm{N}-\mathrm{NH}_{4}^{+}$ (amônia) no solo (UNLU et al., 1999). De preferência, é preciso sincronizar melhor a liberação de $\mathrm{N}$ pelos resíduos, com as fases de maior requerimento nutricional das culturas. É possível alcançar essa sincronização por meio de modelos não lineares que descrevem a mineralização do $\mathrm{N}$ em função do tempo. Estes modelos apresentam leis

\footnotetext{
'Doutor em Estatística e Experimentação Agropecuária - Faculdade de Ciências Integradas do Pontal - Universidade Federal de Uberlândia/UFU Avenida José João Dib, 2545 - Progresso - 38302-000 - Ituiutaba, MG - janser@pontal.ufu.br

Engenheiro Agrônomo, Doutor em Estatística e Experimentação Agropecuária, Professor Titular - Departamento de Ciências Exatas/DEX - Universidade Federal de Lavras/UFLA - Cx. P. 3037 - 37200-000 - Lavras, MG - joamuniz@ufla.br

3Professora Adjunta do Departamento de Ciências Exatas/UFLA, CP 37 - 37200-000 - Lavras/MG, email: safadi@ufla.br.

${ }^{4}$ Professor Adjunto do Departamento de Ciência do Solo/UFLA, CP 37 - 37200-000 - Lavras/MG, email: csilva@ufla.br.
} 
de formação em função de parâmetros como: $\mathrm{N}$ potencialmente mineralizável $\left(N_{0}\right)$ e taxas de mineralização. Segundo Pereira et al. (2005), ao se ajustar um modelo não linear em estudos desta natureza consegue-se levantar informações sobre a quantidade de nitrogênio mineralizado e disponibilizado durante o ciclo de uma cultura ao longo do tempo e, também, informações sobre a taxa ou constante de mineralização do nitrogênio.

Conduziu-se este trabalho, com o objetivo de implementar uma ilustração de uso da metodologia bayesiana para predizer as quantidades de $\mathrm{N}$ mineralizados por intermédio de modelos não lineares. Para comprovação dos resultados, utilizaram-se dados experimentais associados à mineralização de $\mathrm{N}$ em Latossolo do sul de Minas Gerais sob efeito da calagem, descritos em Silva et al. (1994). Assim, foram consideradas as quantidades acumuladas de $\mathrm{N}$ mineralizado durante onze tempos de incubação $(1,2,3,4,6,8,10,12,14,21$ e 28 semanas). A acidez do solo foi corrigida visando-se elevar a saturação por bases a $60 \%$, com o pH em água do solo atingindo valores na faixa de 6,0-6,2. A extração do $\mathrm{N}$ mineral (nitrato + amônio) formado durante a incubação foi feita pela lavagem periódica do solo com solução de $\mathrm{CaCl}_{2}$ (Cloreto de Cálcio) 0,01 mol L-1. A quantificação do $\mathrm{N}$ mineralizado foi feita em destilador de arraste de vapores, sendo o $\mathrm{N}$-amônio quantificado após a adição de $\mathrm{MgO}$ (Óxido de Magnésio) no extrato de $\mathrm{CaCl}_{2}$ e, o nitrato, após o uso de liga de Devarda no extrato remanescente. As amostras de solo foram incubadas em condições ambientais do Laboratório de Relação Solo-Planta do DCS/ UFLA, com a temperatura ambiente oscilando entre $21 \mathrm{e}$ $28^{\circ} \mathrm{C}$. O delineamento experimental utilizado foi o inteiramente ao acaso com três repetições. Os modelos não lineares utilizados para descrever a mineralização do nitrogênio no solo em função do tempo, e para ilustrar o procedimento bayesiano estão apresentados na Tabela 1.

A descrição dos termos (parâmetros) nos diversos modelos é a seguinte: $y_{i}$ é o nitrogênio mineralizado até o tempo $t_{i} ; t_{i}$ é o tempo de incubação, $\operatorname{com} i=1, \ldots, n ; \quad N_{0}$ é o nitrogênio potencialmente mineralizável; $N_{1}$ é o nitrogênio facilmente mineralizável; $k, k_{0}$ e $k_{1}$ são taxas ou constantes de mineralização; $b$ é um parâmetro que não tem interpretação biológica (constante qualquer); $e_{i}$ é um vetor de erros com distribuição normal com média zero e variância $\sigma_{e}^{2}$.

No contexto de inferência bayesiana, todos os parâmetros do modelo, são considerados como variáveis aleatórias, conforme o conceito subjetivo de probabilidade. A partir de uma generalização do Teorema de Bayes, informações a priori sobre parâmetros são utilizadas em associação com os dados amostrais, possibilitando uma inferência a posteriori sobre os parâmetros (BOX \& TIAO, 1992). A inferência sobre os parâmetros consiste em determinar a posteriori conjunta do modelo, com o objetivo de encontrar uma distribuição para um parâmetro específico, chamada distribuição marginal de $\theta_{i}$. Para isso, integra-se a posteriori conjunta em relação aos outros parâmetros do modelo. Porém, muitas vezes a forma analítica da marginal é complexa e multidimensional ou até mesmo impossível de ser calculada analiticamente. Nesse caso, utilizam-se métodos numéricos iterativos como método de Monte Carlo via Cadeias de Markov, (MCMC) para gerar valores de uma distribuição condicional a posteriori para cada parâmetro (SORENSEN, 1996). Dentre os métodos de simulação que fazem o uso de cadeias de Markov destacam-se o Amostrador de Gibbs e o Metropolis Hastings (HASTINGS, 1970). O Amostrador de Gibbs é indicado quando as distribuições condicionais completas são identificáveis (CASELLA \& GEORGE, 1993). Caso isso não ocorra, pode-se utilizar o algoritmo de "Metropolis Hastings" (HASTINGS, 1970).

Neste trabalho, a monitoração da convergência das cadeias foi realizada por meio do critério de Geweke (1992) e Raftery \& Lewis (1992). A regra de decisão do critério de

Tabela 1 - Modelos não lineares que descrevem a relação de mineralização de nitrogênio no solo em função do tempo (semanas).

\begin{tabular}{llc}
\hline \multicolumn{1}{c}{ Modelo } & Equação & Referência \\
\hline (1) Stanford e Smith & $y_{i}=N_{0}\left[1-\exp \left(-k t_{i}\right)\right]+e_{i}$ & Stanford \& Smith (1972). \\
(2) Marion & $y_{i}=N_{0}\left[1-\exp \left(-k t_{i}^{b}\right)\right]+e_{i}$ & Marion et al. (1981). \\
(3) Cabrera & $y_{i}=N_{1}\left[1-\exp \left(-k_{1} t_{i}\right)\right]+k_{0} t_{i}+e_{i}$ Cabrera (1993).
\end{tabular}


Raftery \& Lewis (1992) é baseada no fator de dependência. Se o fator de dependência for maior que 5 , pode-se concluir que a cadeia não atingiu convergência. A regra de decisão do critério de Geweke (1992) é baseada na estimativa do p-valor. Se o p-valor for menor que um nível de significância adotado pelo pesquisador, conclui-se que a cadeia não atingiu convergência. A comparação dos modelos realizou-se por intermédio do Fator de Bayes (BERKHOF et al., 2003) e do Critério de Informação Bayesiano (CARLIN $\&$ LOUIS, 2000). Se o Fator de Bayes (FB) for maior que 1, o modelo 1 apresenta uma probabilidade a posteriori maior, ou seja, o modelo 1 é mais plausível ou verossímil do que o modelo 2, então, opta-se por escolher o modelo 1 para predizer o fenômeno em questão. A regra de decisão baseada no Critério de Informação Bayesiano (BIC) consiste na escolha do modelo que apresentar maior valor de $B I C_{i}$.

Os elementos necessários para uma análise bayesiana dos modelos não lineares (1), (2) e (3) estão descritos a seguir. Primeiramente, para o modelo de Stanford \& Smith, equação (1), supondo que $e_{i} \sim$ iid $N\left(0, \tau_{e}^{-1}\right), \quad \tau_{e}=\left(\sigma_{e}^{2}\right)^{-1}, \quad$ então, verossimilhança pode ser escrita da seguinte forma:

$$
\begin{gathered}
L\left(y \mid N_{0}, k, \tau_{e}, H\right) \propto \tau_{e}^{n / 2} \exp \\
\exp \left\{-\frac{\tau_{e}}{2} \sum_{i=1}^{n}\left\{y_{i}-N_{0}\left[1-\exp \left(-k t_{i}\right)\right]\right\}^{2}\right\}
\end{gathered}
$$

em que $i=1, \ldots, n, y=\left\{y_{1}, y_{2}, \ldots, y_{n}\right\}$ e $H$ são os hiperparâmetros: $\alpha_{e}$ e $\beta_{e}$.

O passo seguinte consiste na definição das distribuições a priori dos parâmetros do modelo (1). De acordo com Sorensen (1996), admitindo que não haja informação prévia a respeito dos parâmetros $N_{0}$ e $k$, definiu-se uma priori do tipo função constante no espaço paramétrico (priori não informativa), ou seja,

$$
p\left(N_{0}, k\right) \propto w
$$

Para a precisão $\tau_{e}$, assumiu-se como priori, uma distribuição Gama com hiperparâmetros $\alpha_{e}$ e $\beta_{e}$, ou seja,

$$
p\left(\tau_{e} \mid \alpha_{e}, \beta_{e}\right) \propto \tau_{e}^{\alpha_{e}-1} \exp \left\{-\beta_{e} \tau_{e}\right\}
$$

Combinando a verossimilhança (4) com as priori (5) e (6), pelo Teorema de Bayes, obtém-se a distribuição a posteriori conjunta do modelo de Stanford \& Smith (1):

$p\left(N_{0}, k, \tau_{e} \mid y, H\right) \propto L\left(y \mid N_{0}, k, \tau_{e}, H\right) p\left(N_{0}, k\right) p\left(\tau_{e} \mid H\right)$

ou seja,

$$
\begin{gathered}
p\left(N_{0}, k, \tau_{e} \mid y, H\right) \propto \tau_{e}^{\frac{n}{2}+\alpha_{e}-1} \times \exp \\
\exp \left\{-\frac{\tau_{e}}{2}\left[2 \beta_{e}+\frac{1}{2} \sum_{i=1}^{n}\left\{y_{i}-N_{0}\left[1-\exp \left(-k t_{i}\right)\right]\right\}^{2}\right]\right\}
\end{gathered}
$$

Analogamente, tem-se que as distribuições a posteriori conjuntas dos modelos de Marion (2) e Cabrera (3), são respectivamente (9) e (10):

$$
\begin{gathered}
p\left(N_{0}, k, b, \tau_{e} \mid y, H\right) \propto \tau_{e}^{\frac{n}{2}+\alpha_{e}-1} \times \exp \\
\exp \left\{-\frac{\tau_{e}}{2}\left[2 \beta_{e}+\sum_{i=1}^{n}\left\{y_{i}-N_{0}\left[1-\exp \left(-k t_{i}^{b}\right)\right]\right\}^{2}\right]\right\} \\
p\left(N_{1}, k_{1}, k_{0}, \tau_{e} \mid y, H\right) \propto \tau_{e}^{\frac{n}{2}+\alpha_{e}-1} \times \exp
\end{gathered}
$$

$\exp \left\{-\frac{\tau_{e}}{2}\left[2 \beta_{e}+\frac{1}{2} \sum_{i=1}^{n}\left\{y_{i}-N_{1}\left[1-\exp \left(-k_{1} t_{i}\right)\right]-k_{0} t_{i}\right\}^{2}\right]\right\}$

A estimação dos parâmetros realizou-se por meio de rotinas elaboradas no software $\mathrm{R}$ e para o procedimento de amostragem utilizou-se o Amostrador de Gibbs e o Metropolis Hastings. No processo foram geradas 450.000 iterações, considerando-se um "burn-in" de 50.000, isto é, as 50.000 primeiras iterações foram descartadas, e um "thin" a cada 100 iterações para assegurar a independência da amostra.

A inferência sobre cada parâmetro dos modelos não lineares (1), (2) e (3) se realiza por meio das distribuições marginais dos parâmetros. Portanto, a partir das posteriori conjuntas, (8), (9) e (10), foram obtidas as distribuições a posteriori condicionais completas para cada parâmetro dos modelos (1), (2) e (3), respectivamente. A implementação do algoritmo do Amostrador de Gibbs, o qual permite obter aproximações das distribuições marginais foi realizada no software R, conforme R Development Core Team (2004).

A obtenção das distribuições condicionais completas dos parâmetros dos modelos de Marion e Cabrera está detalhada no Anexo. Para o modelo de Stanford \& Smith (1972) (1), segundo a sua posteriori conjunta (8), a 
posteriori condicional completa para o parâmetro $N_{0}$, tem como distribuição condicional uma Normal, dada por:

$$
p\left(N_{0} \mid k, \tau_{e}, y, H\right) \propto \exp \left\{-\frac{\tau_{e}}{2} \sum_{i=1}^{n}\left\{y_{i}-N_{0}\left[1-\exp \left(-k t_{i}\right)\right]\right\}^{2}\right\}
$$

Considere $a_{i}=1-\exp \left(k t_{i}\right)$ e $a$ como vetor dos elementos $a_{i}$ para o tempo $i, \operatorname{com} i=1,2, \ldots, n$ e $y=\left\{y_{1}, y_{2}, \ldots, y_{n}\right\}$ pode-se definir (11) como:

$$
\begin{gathered}
p\left(N_{0} \mid k, \tau_{e}, y, H\right) \propto \exp \\
\exp \left\{-\frac{\tau_{e}}{2}\left[N_{0}-\left(a^{\prime} a\right)^{-1} a^{\prime} y\right]^{\prime}\left(a^{\prime} a\right)\left[N_{0}-\left(a^{\prime} a\right)^{-1} a^{\prime} y\right]\right\}
\end{gathered}
$$

isto é,

$$
\begin{gathered}
\left(N_{0} \mid k, \tau_{e}, y, H\right) \sim N\left(\left(a^{\prime} a\right)^{-1} a^{\prime} y, \tau_{e}\left(a^{\prime} a\right)^{-1}\right) \\
\text { em que } a^{\prime} a=\sum_{i}\left[1-\exp \left(-k t_{i}\right)\right]^{2} \mathrm{e} \\
\left(a^{\prime} a\right)^{-1} a^{\prime} y=\left\{\sum_{i}\left[1-\exp \left(-k t_{i}\right)\right] y_{i}\right\} / \sum_{i}\left[1-\exp \left(-k t_{i}\right)\right]^{2}
\end{gathered}
$$

Logo, a posteriori condicional completa para $N_{0}$ pode ser reescrita da seguinte forma:

$\left(N_{0} \mid k, \tau_{e}, y, H\right) \sim N\left(\frac{\sum_{i}\left[1-\exp \left(-k t_{i}\right)\right] y_{i}}{\sum_{i}\left[1-\exp \left(-k t_{i}\right)\right]^{2}}, \frac{\tau_{e}}{\sum_{i}\left[1-\exp \left(-k t_{i}\right)\right]^{2}}\right)$
Para o parâmetro $k$ do modelo (1), tem-se:

$$
\begin{gathered}
p\left(k \mid N_{0}, \tau_{e}, y, H\right) \propto \exp \left\{-\frac{\tau_{e}}{2} \sum_{i=1}^{n}\left\{y_{i}-N_{0}\left[1-\exp \left(-k t_{i}\right)\right]\right\}^{2}\right\} \\
\text { E, finalmente, para o parâmetro } \tau_{e}, \text { tem-se: }
\end{gathered}
$$

$$
p\left(\tau_{e} \mid N_{0}, k, y, H\right) \propto \tau_{e}^{\frac{n}{2}+\alpha_{e}-1} \times \exp \left\{-\frac{\tau_{e}}{2}\left[2 \beta_{e}+\sum_{i=1}^{n}\{\right.\right.
$$

$$
\left.\left.\left\{y_{i}-N_{0}\left[1-\exp \left(-k t_{i}\right)\right]\right\}^{2}\right]\right\}
$$

ou seja, a distribuição a posteriori condicional completa para $\tau_{e}$ é uma Gama:

$$
\begin{gathered}
\left(\tau_{e} \mid N_{0}, k, y, H\right) \sim G\left(\alpha_{e}^{*}=\frac{n}{2}+\alpha_{e}, \beta_{e}^{*}=\beta_{e}+\frac{1}{2} \sum_{i=1}^{n}\{\right. \\
\left.\left\{y_{i}-N_{0}\left[1-\exp \left(-k t_{i}\right)\right]\right\}^{2}\right)
\end{gathered}
$$

Com base na Tabela 2, verifica-se que o critério de Geweke (1992) não apresentou nenhuma evidência contra a convergência dos parâmetros dos modelos, ao nível de $5 \%$, e com relação ao critério de Raftery \& Lewis (1992) observa-se que os fatores de dependência para cada parâmetro dos modelos são inferiores a "cinco", o que caracteriza a convergência das cadeias.

De acordo com a Tabela 3, pode-se afirmar que as estimativas dos intervalos HPD mostraram-se adequadas ao

Tabela 2 - Fator de Dependência do critério de Raftery \& Lewis (1992) e p-valor do critério de Geweke (1992) dos modelos (1), (2) e (3).

\begin{tabular}{cccc}
\hline Modelo & Parâmetro & FD $^{(1)}$ & Geweke p-valor \\
\hline Stanford \& Smith & $N_{0}$ & 1,0109 & 0,0636 \\
& $k$ & 1,0534 & 0,9899 \\
& $\tau_{e}$ & 0,9907 & 0,9982 \\
\hline Marion & $N_{0}$ & 1,0109 & 0,9565 \\
& $k$ & 2,5056 & 0,9925 \\
& $b$ & 1,0320 & 0,9845 \\
Cabrera & $\tau_{e}$ & 0,9907 & 0,9834 \\
\hline & $N_{1}$ & 1,1439 & 0,8953 \\
& $k_{1}$ & 0,9704 & 0,9991 \\
& $k_{0}$ & 1,0109 & 0,9552 \\
\hline
\end{tabular}

${ }^{1}$ FD: Fator de Dependência do critério de Raftery \& Lewis (1992). 
Tabela 3 - Média e desvio padrão a posteriori dos parâmetros dos modelos (1), (2) e (3), com seus respectivos intervalos HPD 95\%, erro de Monte Carlo (Erro MC).

\begin{tabular}{|c|c|c|c|c|c|c|}
\hline \multirow{2}{*}{ Modelo } & \multirow{2}{*}{ Par. ${ }^{(1)}$} & \multirow{2}{*}{$\begin{array}{c}\text { Média } \\
\text { Posteriori }\end{array}$} & \multicolumn{2}{|c|}{$\mathrm{HPD}^{(2)} 95 \%$} & \multirow{2}{*}{$\begin{array}{l}\text { Desvio } \\
\text { Padrão }\end{array}$} & \multirow{2}{*}{ Erro $\mathrm{MC}^{(4)}$} \\
\hline & & & $\mathrm{LI}^{(3)}$ & $\mathrm{LS}^{(3)}$ & & \\
\hline \multirow{3}{*}{$\begin{array}{c}\text { Stanford \& } \\
\text { Smith }\end{array}$} & $N_{0}$ & 150,8158 & 148,2554 & 153,5245 & 1,3606 & 0,01953957 \\
\hline & $k$ & 0,1933 & 0,1806 & 0,2046 & 0,0062 & 0,01581145 \\
\hline & $\tau_{e}$ & 0,0267 & 0,0093 & 0,0486 & 0,0106 & 0,01581159 \\
\hline \multirow{4}{*}{ Marion } & $N_{0}$ & 149,4726 & 145,8477 & 152,8670 & 1,7807 & 0,02470511 \\
\hline & $k$ & 0,1932 & 0,1812 & 0,2052 & 0,0062 & 0,01581146 \\
\hline & $b$ & 1,0171 & 1,0000 & 1,0497 & 0,0158 & 0,01581183 \\
\hline & $\tau_{e}$ & 0,0245 & 0,0075 & 0,0435 & 0,0101 & 0,01581158 \\
\hline \multirow{4}{*}{ Cabrera } & $N_{1}$ & 256,8945 & 247,5555 & 265,7531 & 4,6515 & 0,2484605 \\
\hline & $k_{1}$ & 0,1009 & 0,0963 & 0,1057 & 0,0024 & 0,0158114 \\
\hline & $k_{0}$ & $-3,3107$ & $-3,5531$ & $-3,0576$ & 0,1241 & 0,01584023 \\
\hline & $\tau_{e}$ & 0,0446 & 0,0141 & 0,0776 & 0,0173 & 0,01581184 \\
\hline
\end{tabular}

${ }^{1}$ Par.: Parâmetros dos modelos; ${ }^{2}$ HPD: intervalo de máxima densidade a posteriori; ${ }^{3}$ LI e LS: limite inferior e superior do intervalo HPD $;{ }^{4}$ Erro MC: Erro de Monte Carlo.

ajuste dos modelos, pois foram obtidas a partir da distribuição empírica dos parâmetros, e observa-se também que todos os parâmetros dos modelos (1), (2) e (3) foram significativos, pois seus respectivos intervalos HPD não continham o valor zero em sua extensão. Nota-se ainda que os Erros de Monte Carlo apresentaram valores baixos, confirmando a boa qualidade das estimativas dos parâmetros.

Por intermédio do Fator de Bayes (Tabela 4), compararam-se os modelos de Stanford \& Smith vs Cabrera e Marion vs Cabrera os resultados foram, 0,0507 e 0,0307, respectivamente. Com isso, pode-se afirmar que o modelo de Cabrera se mostrou melhor que os demais no ajuste aos dados. O Fator de Bayes para a comparação Stanford \& Smith vs Marion apresentou resultado de 1,6504, isto é, o modelo de Stanford \& Smith é mais plausível ou verossímil que o modelo de Marion. Portanto, o modelo que melhor se ajustou aos dados de mineralização de nitrogênio foi o modelo de Cabrera, sendo seguido pelo modelo de Stanford $\&$ Smith e por último o de Marion.

Tabela 4 - Fator de Bayes dos modelos (1), (2) e (3).

\begin{tabular}{cc}
\hline Modelos Confrontados & FB $^{(1)}$ \\
\hline Stanford \& Smith vs Marion & 1,6504 \\
Stanford \& Smith vs Cabrera & 0,0507 \\
Marion vs Cabrera & 0,0307 \\
\hline
\end{tabular}

${ }^{1} \mathrm{FB}$ : Fator de Bayes.
A comparação de modelos por meio do Critério de Informação Bayesiano (BIC) consiste na escolha do modelo que apresentar o maior valor de BIC. Portanto, com base nos resultados apresentados na Tabela 5, o modelo que melhor se ajustou ao conjunto de dados de mineralização do nitrogênio foi o modelo de Cabrera, sendo seguido pelo modelo de Stanford \& Smith e por último o de Marion.

Tabela 5 - Critério de Informação Bayesiano dos modelos (1), (2) e (3).

\begin{tabular}{cc}
\hline Modelos & $B I C^{(1)}$ \\
\hline Stanford \& Smith & $-58,77588$ \\
Marion & $-62,17582$ \\
Cabrera & $-55,20933$ \\
\hline
\end{tabular}

${ }^{1}$ BIC : Critério de Informação Bayesiano.

A inferência bayesiana é uma metodologia de grande potencial para o estudo da mineralização do nitrogênio, pois considera a incerteza existente sobre todos os parâmetros dos modelos estudados, além de possibilitar a inclusão de informações por meio do uso de distribuições a priori informativas. Nas condições em que foram realizados os estudos (tipo de solo, região, presença de calagem), o modelo que proporcionou melhor qualidade de ajuste ao conjunto de dados de mineralização de nitrogênio foi o modelo de Cabrera, tanto utilizando o Fator de Bayes quanto o Critério de Informação Bayesiano. 


\section{AGRADECIMENTOS}

À Coordenação de Aperfeiçoamento de Pessoal de Nível Superior - CAPES, pela bolsa de estudo concedida e à Fundação de Amparo à Pesquisa do Estado de Minas Gerais pelo financiamento do projeto de pesquisa.

\section{REFERÊNCIAS BIBLIOGRÁFICAS}

BERKHOF, J.; MECHELEN, I. V.; GELMAN, A. A bayesian approach to the selection and testing of mixture models. Statistica Sinica, [S.1.], v. 13, p. 423-442, 2003.

BOX, G. E. P.; TIAO, G. C. Bayesian inference in statistical analysis. New York: J. Wiley \& Sons, 1992. $588 \mathrm{p}$.

CABRERA, M. L. Modeling the flush of nitrogen mineralization caused by drying and rewetting soils. Soil Science Society of America Journal, Madison, v. 57, p. 63-66, 1993.

CASELLA, G.; GEORGE, E. Explaning the gibbs sampler. The American Statitician, Washington, v. 46, p. 167-174, 1993.

CARLIN, B. P.; LOUIS, T. A. Bayes and empirical bayes methods for data analysis. 2. ed. London: Chapman and Hall, 2000. 419 p.

FERGUSON, R. B.; SHAPIRO, C. A.; HERGERT, G. W.; KRANZ, W. L.; KLOCKE, N. L.; KRULL, D. H. Nitrogen and irrigation management practices to minimize nitrate leaching from irrigate corn. Journal of Production Agriculture, Madison, v. 4, p. 186-192, 1991.

GEWEKE, J. Evaluating the accuracy of sampling-based approaches to the calculation of posterior moments. In: BERNARDO, J. M.; BERGER, J. O.; DAWID, A. P.;
SMITH, A. F. M. (Eds.). Bayesian statistics 4. New York: Oxford University, 1992. 526 p.

HASTINGS, W. K. Monte carlo sampling methods using Markov chains and their applications. Biometrika, London, v. 57, n. 1, p. 97-109, 1970.

MARION, G. M.; KUMMEROW, J.; MILLER, P. C. Predicting nitrogen mineralization in chaparral soils. Soil Science Society of America Journal, Madison, v. 45, p. 956-961, 1981.

PEREIRA, J. M.; MUNIZ, J. A.; SILVA, C. A. Nonlinear models to predict nitrogen mineralization in a Oxisol. Scientia Agricola, Piracicaba, v. 62, n. 4, p. 395-400, 2005.

RAFTERY, A. L.; LEWIS, S. Comment: one long run with diagnostics: implementation strategies for Markov chain Monte Carlo. Statistical Science, Hayward, v. 7, n. 4, p. 493-497, 1992.

\section{R DEVELOPMENT CORE TEAM. The R manuals:} current version: 1.9.1. 2004. Disponível em: <http:// LWWW.r-project.org'>. Acesso em: 10 out. 2006.

SILVA, C. A.; VALE, F. R.; GUILHERME, L. R. Efeito da calagem na mineralização do nitrogênio em solos de Minas Gerais. Revista Brasileira Ciência do Solo, Campinas, v. 18, p. 471-476, 1994.

SORENSEN, D. Gibbs sampling in quantitative genetics. Foulun: [s.n.], 1996. 186 p.

STANFORD, G.; SMITH, S. J. Nitrogen mineralization potentials of soil. Soil Science of America Journal, Madison, v. 36, p. 465-472, 1972.

UNLU, K.; OZENIRLER, G.; YURTERI, C. Nitrogen fertilizer leaching from cropped and irrigated sandy soil in Central Turkey. European Journal of Soil Science, Oxford, v. 50, n. 4, p. 609-620, 1999. 